\title{
Human Saliva as a Diagnostic Specimen for Early Detection of Inflammatory Biomarkers by Real-Time RT-PCR
}

\author{
Nourin Shakeeb, ${ }^{1}$ Prashanth Varkey, ${ }^{2}$ and Amita Ajit $\mathbb{D}^{1,3}$
}

(Received February 15, 2021; accepted May 20, 2021)

Abstract-Nowadays human saliva is more frequently studied as a non-invasive, stress-free, and preferable diagnostic material than blood. Supporting evidences acknowledge saliva as a mirror that reflects the body's physical state. Numerous studies have also demonstrated the presence and use of RNA derived from saliva in the early diagnosis of disease by real-time reverse transcriptase polymerase chain reaction (RT-PCR). Assessing the host inflammatory response in patients and its resolution at an early stage can serve as a prognostic and predictive method in determining therapeutic response or disease progression. In this context, the potential of saliva as a specimen to diagnose early inflammatory biomarkers using RT-PCR seems fascinating and useful. Here, we review inflammatory biomarkers within the saliva, focusing on early detection of these biomarkers using RT-PCR and the factors influencing the quality of saliva specimen.

KEY WORDS: Human saliva; Molecular diagnostics; RT-PCR; Biomarkers; Inflammation.

\section{INTRODUCTION}

Saliva is a biofluid with clinical significance that can be easily collected and stored and is proved to be ideal for early detection of systemic diseases [29]. As saliva is a repository of multiple biomarkers, it can be used in rapid

Nourin Shakeeb and Amita Ajit contributed equally to this work.

\footnotetext{
${ }^{1}$ Research and Development, Zum Heilen Diagnostic and Therapeutics Pvt. Ltd, Office No. 12/1543-C, SB Center, 2nd Floor, Museum Road, Thrissur, Kerala 680020, India

${ }^{2}$ Jubilee Centre for Medical Research, Jubilee Mission Medical College \& Research Institute, P.B.No.737, Thrissur, Kerala 680005, India

${ }^{3}$ To whom correspondence should be addressed at Research and Development, Zum Heilen Diagnostic and Therapeutics Pvt. Ltd, Office No. 12/1543-C, SB Center, 2nd Floor, Museum Road, Thrissur, Kerala 680020, India. E-mail: dr.amitaajit@ gmail.com
}

tests, point-of-care (POC) devices, or other standard clinical laboratory operations. Molecular diagnostics using saliva has advanced into a sophisticated and established science, and now it is renowned as a central player in clinical areas and biomedical technology [29]. Saliva collection being a relatively stress-free and non-invasive process can be regarded as a potential alternative diagnostic fluid in young individuals especially infants and toddlers. Currently, saliva is used as a specimen for clinical assessment of hormonal perturbations, detection of HIV antibodies, DNA analysis, drug testing, and alcohol screening. However, saliva is not yet considered as an established analytical specimen due to the inadequate information about the salivary biochemical composition and its interrelationship with plasma levels [10]. Among the currently available molecular analytical tools, RT-PCR serves at the 
gene level of analysis and is proved to be a very evident method for early detection of biomarkers in systemic diseases and its rectification [55]. RT-PCR technique is also regarded as the gold standard for gene-level analysis as it amplifies the target biomarker a billion times thereby facilitating ease in detection even at a very early stage of gene expression [19, 27, 33]. Accordingly, the application of RT-PCR in early salivary diagnostics seems promising and of great clinical interest. In the latest years, one of the many important scientific discoveries in medical research involved the awareness that inflammation plays an inevitable role in many health disorders leading to considerable morbidity and early mortality [47]. Several psychiatric conditions, such as anxiety, bipolar and unipolar depression, posttraumatic stress disorder, and schizophrenia, as well as many physical disease condition such as cardiovascular diseases, respiratory illness, asthma, rheumatoid arthritis, obesity, diabetes, osteoporosis, certain cancers, fatty liver, organ disorders [6], stroke, Alzheimer's disease, physical injuries, and infectious diseases, show considerable inflammatory responses as depicted in Fig. 1. A list of predominant inflammatory markers associated with chronic diseases is also given in Table 1. Hence, the understanding that inflammation is responsible for promoting poor health, and when and how we can intervene to reduce inflammation-related disease risk, should thus be a top scientific priority [14, 28, 47]. The possibility to identify biomarkers in saliva at an early stage could address the clinical need to avail information on inflammation for improved disease diagnosis and effective intervention of therapy and to contribute to the development of new targeted therapeutic drugs. In this perspective, we describe current findings and put forth some promising avenues for future research and intervention on the use of saliva for the assessment of inflammatory response.

\section{METHODOLOGY}

The present review was conducted by comprehensive literature search. The literature search was performed using PubMed, Science Direct, Elsevier, Hindawi, Springer, and Biomed Central databases from 1996 to 2021, by following search strategy: ("saliva" AND "diagnostic specimen") OR ("inflammatory biomarkers" AND "saliva") OR ("RTPCR analysis of inflammatory markers in saliva"). Searches were also done among the reference lists from suitable reviews or original articles to identify further relevant literature. Additional information was also obtained from trusted websites and handbooks.

\section{SALIVA AS A DIAGNOSTIC SPECIMEN}

In recent years, there has been increasing interest in the use of saliva as a diagnostic tool. Collection of patient saliva is easy and non-invasive and requires less stress than any other body fluid. Oral fluid sampling promotes lowcost storage [40]. Moreover, analysis of saliva makes frequent monitoring easy especially in infants, children, and elderly patients, as well as in circumstances where urine or blood sampling is impractical. Additionally, saliva appears fascinating for diagnostic purposes owing to its linkage with traditional biochemical parameters that appear in various forms [8]. The presence of specific proteomic content in human saliva permits researchers to analyze and explore different assays for possible identification of novel biomarkers that can be correlated with the overall health status of a patient [40].

The Saliva Proteome Knowledge Base (http:// www.skb.ucla.edu) is the first-ever database that comprises all the identified proteomic data to date making it readily accessible to the public. The techniques used by scientific researchers to carry out the elucidation of this proteomic data from saliva are nuclear magnetic resonance, capillary electrophoresis, gel electrophoresis, immunoassay, MS, and LC [54]. With this great discovery, scientists have coined the term "salivaomics." Salivaomics highlights all the different types of technologies used for extracting the data about potential salivary biomarkers such as transcriptomics, genomics, microRNA (miRNA) proteomics, and metabonomics [54]. With respect to saliva as a diagnostic specimen, its use in the assessment of many systemic diseases such as autoimmune diseases, cardiovascular diseases, disease of the adrenal cortex, genetic disorders such as cystic fibrosis and ectodermal dysplasia, and infections of bacteria and fungi is reported [30]. In a study by Boppana et al., use of saliva from infants having CMV (cytomegalovirus) infection, congenital infection, and a non-genetic cause of sensorineural hearing loss reported early screening of CMV through RT-PCR [4]. Another study done in patients with oral squamous cell carcinoma (OSCC) from 2015 to 2017 revealed that mRNA levels of NAB2, CYP27A1, COL3A1, MAOB, NPIPB4, and SIAE were significantly less in the saliva of oral cancer patients by performing real-time PCR. The results advocate the use of saliva as a good and stable specimen for early diagnosis and prognosis of chronic oral diseases [35]. These findings 


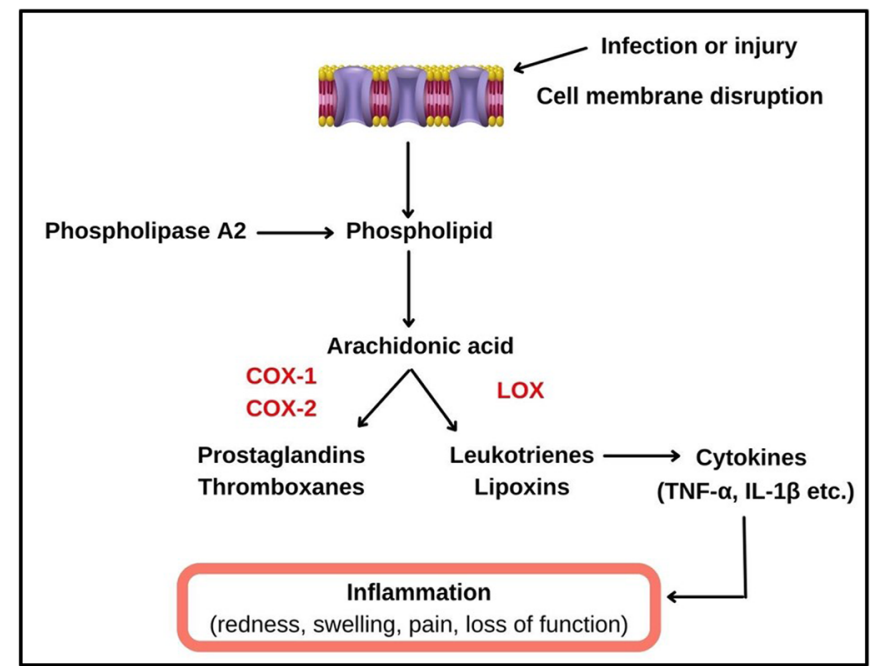

Fig. 1. Mechanism of inflammatory cascade: In the presence of a perceived threat or infection, the cell membrane disrupts and the enzyme phospholipase A2 acts on the phospholipid layer, hydrolysing the esterified arachidonic acid [51]. This polyunsaturated fatty acid metabolizes via enzymes such as cyclooxygenase (COX-1 and COX-2) and lipoxygenase to produce eicosanoids such as prostaglandins, leukotrienes, thromboxanes, and lipoxins. Leukotrines further instigates the production of inflammatory cytokines such as IL-6, IL-1 $\beta$, and TNF- $\alpha$ causing inflammation [52].

thus support the use of saliva as a potential specimen capable of being analyzed using various sophisticated molecular biology techniques such as RT-PCR. Use of saliva for clinical diagnosis also poses several advantages over the use of blood or any other biofluid, such as the following: (i) reduction in healthcare costs: saliva collection does not require specialized technicians; (ii) anticoagulation treatment after collection can be avoided as they do not clot; (iii) much more safer than other traditional specimens (e.g., blood) as there are low chances of disease transmission, for example, infectivity of HIV is inhibited by certain factors in salivary secretions; (iv) molecular activity remains almost constant even after sampling, hence they are "quiescent"; (v) they are relatively clean as they contain few interfering proteins; and (vi) saliva proves that it can be used as a specimen for point-of-care devices, where patients can collect the sample at their homes [39].
However, as a diagnostic specimen, several challenges have been reported in recent years which include the essential need for sensitive biomarker detection systems, need for an apt collection method that does not influence its composition, and the variations due to the diurnal/circadian rhythm. Despite these challenges, saliva is still gaining attention owing to its notable advantages and its great deal of potential biomarkers that range from changes in biochemical, DNA, RNA, and proteins in the oral environment [40].

\section{SCREENING OF INFLAMMATORY BIOMARKERS IN SALIVA}

The National Institutes of Health Biomarkers Definitions Working Group defines a biomarker as a

Table 1. List of inflammatory markers associated with common chronic diseases

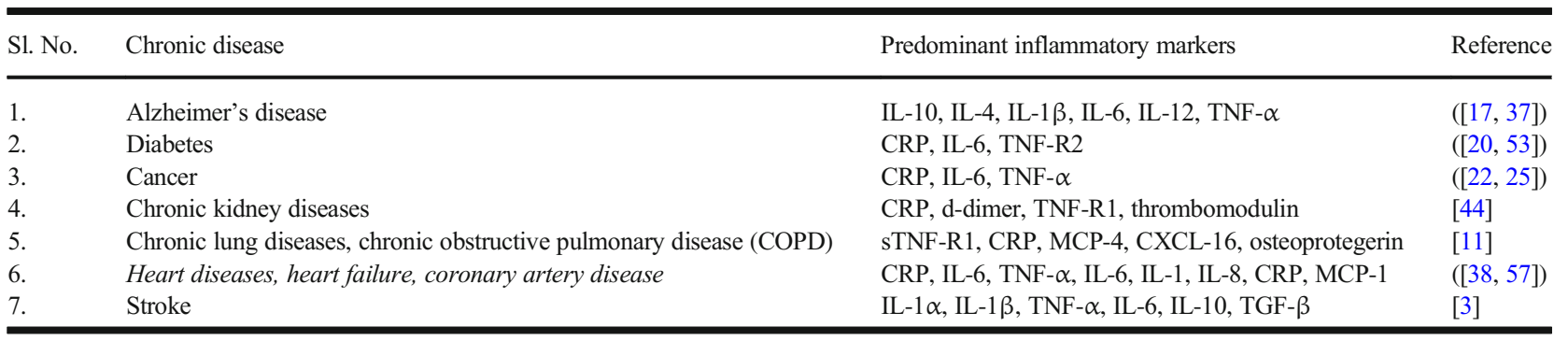


characteristic that objectively measures and evaluates the state of normal biological, pharmacological, or pathogenic response to a therapy [39]. In the last two decades, various inflammatory biomarkers have been discovered such as tumor necrosis factor (TNF), interleukin-1 (IL-1), interleukin-6 (IL-6), arachidonate 5-lipoxygenase (5LOX), prostaglandin-endoperoxide synthase 2 (COX-2), chemokines, vascular endothelial growth factor (VEGF), matrix metalloproteinases (MMPs), twist-related protein (TWIST), and cell surface adhesion molecules. These biomarkers are reported to be detected in biological specimens such as blood, urine, hair, feces, cerebrospinal fluid, sputum/saliva, and body tissues [39]. Protein level detection of salivary markers has always been the gold standard in the diagnostics industry. A recent study reported the detection of acute-phase proteins and cytokines from saliva. Acutephase proteins, predominantly C-reactive proteins (CRPs), aid in producing pro-inflammatory cytokines in response to infection [49]. Salivary CRP levels can predict myocardial infarction and cardiovascular disease, and hence, it may be a particularly health-relevant biomarker of inflammation [36]. Some cytokines such as IL-6, TNF- $\alpha$, and IL$1 \beta$ are locally produced in the mouth and also get filtered into the whole saliva from the oral mucosa transudate, gingival fold, salivary glands, and mucus from the nasal cavity [10]. Even though some studies have reported that the levels of many cytokines are much lower in saliva than in blood, there is a growing literature suggesting that certain markers, such as salivary IL-1 $\beta$ may be detected more in saliva than in blood [34].

Even though saliva can be used as a diagnostic specimen in almost all systemic diseases, it proves to be more efficient when different oral pathologies need to be diagnosed. As saliva is the major biofluid in the mouth, it is with great certainty that an investigator can use it as a tool for assessing the expression of inflammatory markers. Oral diseases such as periodontitis (PD), primary Sjögren's syndrome (pSS), oral lichen planus (OLP), periimplantitis, oral leukoplaki, and medication-related osteonecrosis of the jaw (MRONJ) use saliva as a tool to diagnose as well as to prognose the disease severity [31]. TNF- $\alpha$, IL1 $\beta$, and PGE2 showed increased expression in patients with PD; this suggests their potential use as biomarkers for diagnosis and prognosis. Similarly, in patients with pSS, there was an increased expression of S100A protein in saliva, which directly relates to IL-12 production [31]. Many authors have pondered the potential of interleukins (ILs) as salivary markers for diagnosing and monitoring the progression of oral leukoplakia. It is reported that IL6, IL8, and TNF- $\alpha$ levels are elevated in patients affected with oral leukoplakia [31, 43]. Singh et al. validated IL-1 $\beta$, IL-8, and Lgals3bp (using ELISA) for detecting oral squamous cell carcinoma (OSCC) in an Indian population. IL-1 $\beta$ and IL- 8 were expressed highly in OSCC patients than those in controls. Lgals3bp significantly discriminated the early stage of OSCC (stages I-II) [46]. Another study highlighted the detection of salivary interleukin-6 (IL-6) using enzyme-linked immunosorbent assay (ELISA) in children with early childhood caries (ECC) post treatment. The reduction in the mean level of salivary IL- 6 before and 3 months post treatment indicated post-dental rehabilitation of children with ECC [32].

Nijawoski and Surdacka et al. reported certain salivary biomarkers which can diagnose inflammatory bowel diseases. This review highlighted that salivary IL-6 concentrations were significantly higher in Crohn's disease (CD) patients. CD is an inflammatory bowel disease which causes inflammation of the digestive tract, leading to severe diarrhea, abdominal pain, weight loss, fatigue, and malnutrition (Nijawoski \& Surdacka et al., 2020). The world-shaking pandemic COVID-19 also agrees to the effective use of saliva as a diagnostic specimen to replace and overcome the difficulties in using nasopharyngeal or oropharyngeal swabs (sample collection may cause bleeding and patient discomfort), thus helping to monitor patients with various degree of disease severity, including the asymptomatic carriers [42]. Recent studies have pointed out increased plasma levels of IL-6, IL-7, IL-2, granulocyte colony-stimulating factor (GSCF), IL-10, tumor necrosis factor- $\alpha$, INF- $\gamma$-inducible protein-10 (IP-10), macrophage inflammatory protein-1A, and macrophage chemotactic protein 1 in severe COVID-19 cases, indicating that the inflammatory response mediated by cytokine release is critical in the progression of COVID-19. These cytokines and chemokines can also be detected in saliva, aiding in hassle-free sample collection [42]. Similarly, TNF- $\alpha$, IL$1 \beta$, IL-6, IL-10, IL-12p70, and IL-8 can be used to diagnose cerebral palsy patients with gingivitis. The expression profiles were recorded before and after periodontal treatment [56].

Bloom et al. suggest that salivary IL-6 is a potential non-invasive biomarker for Huntington's disease (HD) symptoms, which is a progressive and inherited neurodegenerative disorder typically affecting adults of age 30-40 [9]. The advent of an effective and reliable salivary biomarker would undoubtedly meet the urgent need for a noninvasive means of detecting and monitoring HD disease progression [9]. Another recent report states that the best marker combination which includes inflammatory cytokines that differentiated active TB (tuberculosis) from 
healthy controls were fractalkine + IP- $10+$ IL- $1 \alpha+$ VEGF in saliva [12]. The higher salivary concentrations of IL17A, IL-17F, and TNF- $\alpha$ were significantly associated with disease advancement in patients with oral and oropharyngeal cancer. Lower levels of IL-17A indicated oral cavity colonization with aerobic bacteria. Contrastingly, higher concentration of TNF- $\alpha$ indicated positive aerobic culture of oral swabs from patients. The result suggested that IL-17F, IL-17A, and TNF- $\alpha$ measured in the saliva may be a potential biomarker for oral and oropharyngeal cancer [58].

It may be noted that in the above-mentioned studies, the inflammatory biomarkers were detected and reported to be quantified using protein level assays. These findings drive possible initiatives to the potential analysis of these markers at a genetic level which could provide a much earlier diagnosis. Accordingly, this calls for an urgent need to investigate the presence of inflammatory markers at a gene level using RT-PCR in saliva. Even though less research has taken place in this area, there are some impactful studies done by investigators which encourage and emphasize gene-level detection of salivary markers. In a study by Gomes et al., expression of inflammatory biomarkers such as IFN- $\gamma$ and TNF- $\alpha$ in whole saliva was screened using RT-PCR to assess chronic moderate to chronic severe periodontal disease in type II diabetic patients [15]. Results indicated high levels of IFN- $\gamma$ mRNA for $15.4 \%$ from the chronic moderate periodontal disease group, suggesting that the cellular immune response is suppressed in these patients and 50\% in samples from chronic severe periodontal disease patients. The detection of mRNA expression for TNF- $\alpha$ was seen in $62 \%$ of patients from the chronic moderate periodontal disease group and $60 \%$ from the chronic severe periodontal disease group suggesting that these cytokines play an active role in maintaining the inflammatory response [15]. The most common conditions prevailing in the human population, such as Asthma, can also be diagnosed and monitored using salivary markers such as amphiregulin (AREG) [18]. In this study, plasma and salivary AREG protein concentrations were analyzed using ELISA and the circulating AREG mRNA was analyzed using RT-qPCR. Asthmatic patients showed high AREG protein levels in blood and saliva compared to healthy subjects; also, there was a high AREG mRNA expression in saliva [18].

In another study performed by Cheng et al., the expression profiles of inflammatory genes in patients with head trauma were reported. This study involved 19 subjects; seven healthy controls with no history of head trauma, six patients from an outpatient concussion clinic diagnosed with concussion injury from outpatient clinic (CCPT), and 6 patients from the emergency department (EDPT) who received treatment for traumatic brain injury (TBI) within $24 \mathrm{~h}$. Analysis of the inflammatory genes in salivary extracellular vesicles (EV) of participants was done by real-time PCR using the TaqMan Human Inflammation array. The expression analysis disclosed nine upregulated genes in emergency department patients (LTA4H, LOX5, CASP1, IL2RG, ANXA3, ITGAM, ITGB2, TNFRSF1A, and MAPK14) and 13 upregulated genes in CCPT compared with healthy participants (ADRB1, BDKRB1, LTB4R2, LTB4R, PTAFR, CYSLTR1, ADRB2, HRH1, HRH2, CES1, KLK1, MC2R, and PTGER3). A unique profile was observed in each patient group. Comparison between these groups indicated that significant expression change was shown by 15 inflammation-related genes, thus revealing that inflammatory biomarkers are a potential source for diagnosis of traumatic brain injury and in assessing the severity of the condition [7]. Another research study highlighted that 27 inflammatory cytokines detected in blood, such as IL-1 $\beta$, IL-2, IL-9, IL-10, IL13, IL-17, IL-12, and eotaxin, were detected in healthy adult saliva as well. A correlation study used 16 inflammatory biomarkers in healthy and depressed adolescents which showed a higher detection rate of 14 out of 16 biomarkers in more than $80 \%$ of saliva samples except for IL-10 and INF- $\alpha 2$ [5]. An interesting study by Al-Rawi et al. proved salivary microRNAs (miRNAs) to be an efficient biomarker for detecting periodontal disease among diabetic and non-diabetic patients. This study validated four miRNAs, namely, miRNA 155, $146 \mathrm{a} / \mathrm{b}$, and 203. Results of the cohort study indicated that all the four miRNA expressions were seen high in patients with periodontitis and/or diabetes [1]. Therefore, from these studies, it is evident that by incorporating RT-PCR as a routine diagnostic tool, early diagnosis and effective monitoring of disease severity using saliva can be done as efficiently as possible.

\section{COMPARATIVE ANALYSIS OF SALIVARY BIOMARKERS USING RT-PCR AND PROTEOMIC APPROACHES}

Despite the encouraging number of studies cited earlier that focuses on RT-PCR-mediated detection of biomarkers in saliva, a very limited number of studies have investigated comparative analysis of salivary biomarkers using RT-PCR and proteomic approaches. Moreover, comparative analysis of biomarkers in saliva at the 
transcriptome and proteome levels becomes meaningful only when both RT-PCR and protein analysis are performed within the same individual at the same time period. $\mathrm{S}$. Hu et al. reported that a certain degree of concordance was observed in testing the hypothesis that salivary proteins and their counterpart mRNA co-existed in human saliva (S. [21]). In his study, saliva proteome analysis was performed using "shotgun" and 2-D electrophoresis/mass spectroscopy (2-DE/MS) approaches while saliva transcriptome analysis was performed by microarray followed by validation with RTPCR. On proteomic profiling, 309 salivary proteins were detected in the 1st healthy participant, 282 salivary proteins in the 2nd healthy participant, and 297 salivary proteins in the 3rd healthy participant. Interestingly, the salivary mRNA profiles of the same three participants performed using HG U133A expression arrays yielded 2940, 2521, and 3363 mRNAs in healthy participants 1 , 2 , and 3 respectively. To overcome false negative results in microarray profiling, the "undetectable" mRNAs from participants were validated using RTPCR. Their findings concluded that salivary proteins and their counterpart mRNAs do co-exist and that their transcriptome may be detected by RT-PCR analysis at a higher extent in comparison to proteomics and microarray outcomes (S. [21]). Another study by John et al. examined the detection of 2 specific inflammatory cytokines, IL-6 and IL-8, in the saliva of patients with oral cavity and oropharyngeal squamous cell carcinoma (OSCC) using RT-PCR and ELISA technique. The findings confirmed the mRNA and the protein level results to be concordant [23].

In the literature search, no other comparative studies in similar lines were found using the key words "salivary biomarkers," "comparative analysis," "RTPCR Analysis," "ELISA," "proteomic analysis," and "genomic analysis." Further studies on comparative analysis of salivary biomarker profiles within the same individuals using RT-PCR and proteomic approaches are thus encouraged to validate the superiority of the RT-PCR technique in early detection for disease diagnosis at the clinical level.

\section{FACTORS INFLUENCING DIAGNOSIS IN SALIVA}

Whole saliva is a mixture of saliva secreted in the oral cavity from various glands along with constituents such as bronchial and nasal secretions, tears, food debris gingival crevicular fluid, and bacteria [2]. Numerous factors do influence the diagnostic efficiency in saliva specimen such as the collection method, age and gender of the patient $[2$, 48], preservation or storage, mouth position during saliva collection [2], and how well the biomarkers are expressed in it compared to serum, whole blood, or any other biofluids. Proper sample collection should involve sufficient sample volume, accurate patient identification, and the appropriate type of collection container, i.e., the container must have a design that facilitates easy spitting and accumulation of saliva at the bottom. There are two ways in which saliva can be collected: unstimulated and stimulated [45]. Unstimulated saliva can be collected by drooling the saliva in the mouth for a minute and draining it into a sterile vessel or by suction method or swabbing method. Saliva collected by masticatory action, i.e., by gustatory stimulation by applying acetic acid in the mouth or chewing paraffin wax yields stimulated whole saliva. Stimulated saliva possesses certain disadvantages as the foreign substances that stimulate the saliva modifies the $\mathrm{pH}$ and the water phase of the secretion, resulting in a dilute sample [45]. It is also recommended by Bhattarai et al. that samples may be collected by passive drooling as large amount of saliva can be collected in a short period [2].

Saliva comprises many constituents including water, enzymes, electrolytes, and antimicrobial agents. These constituents can vary or remain stable with age and gender. For example, in elderly individuals, salivary flow rate and calcium were observed to be decreased compared to young individuals. Interestingly, newborns and adults also exhibited differences in their salivary protein profile [13]. The unstimulated saliva secretion was seen higher in healthy men compared to that in women, owing to differences in the gland size and its effect on salivary secretion as females have smaller salivary glands, concluding that there is a gender-dependent secretion. Hence, age groups and gender must be categorized and considered to reduce statistical errors [13]. It is also reported that if the diagnostic test is to be performed immediately, salivary specimens can be stored at room temperature for a duration of 30-90 $\mathrm{min}$ [8]. On the contrary, Thomadaki $\mathrm{K}$ et al. clearly states that if incubation temperature is lowered, then the degradation rate of the salivary proteome is also reduced [50]. Another study reported that repeated freeze-thaw cycles of the salivary specimen should not be entertained [16]. Thus, it is recommended to freeze the secretions at or below $-20^{\circ} \mathrm{C}$ immediately after saliva collection. Specimens can also be stored at $4{ }^{\circ} \mathrm{C}$ to prevent degradation of salivary molecules and microbial growth, but should not be stored for more than $6 \mathrm{~h}$. Specimens can also be stored at $-80^{\circ} \mathrm{C}$ for several 
years with a very low chance of degradation [2]. The different pairs of salivary glands which include parotid glands, sublingual glands, submandibular glands, and numerous other minor salivary glands also influence diagnostic efficiency [2]. Even though the saliva secreted from these glands comprises common components, the concentrations vary from one gland to another. For example, a large number of serous acinar cells are seen in the parotid glands along with that they produce high levels of prolinerich proteins and alpha-amylase, whereas the submandibular gland secretes less alpha-amylase and more mucins than the parotid gland [2]. In addition to salivary origin, salivary components and saliva production also depend on the individual state of being, i.e., the resting state or the stimulated state. For instance, components such as alphaamylase, cortisol, and secretory IgA levels are affected by stimulation [2].

The wide expression of salivary biomarkers is based on the circadian rhythms, and hence, they show an impact on the dynamics and kinetics of these markers [45]. Furthermore, the components in saliva are present at 100-1000-fold lower than the serum levels which hampers its use in routine diagnostic technologies. The specific biomarker panel for diagnostic application requires extensive validation and standardization before implementing it in clinical usage. Thus, formulating corrective actions to overcome the above-mentioned limitations is essential to make saliva a potential diagnostic specimen.

\section{GUIDELINES FOR QUALITY CONTROL IN SALIVARY DIAGNOSTICS}

The probability of sampling error is highly witnessed during saliva collection and processing [2]. In order to avoid such errors, investigators must make use of questionnaire answers to select eligible volunteers. It is always better to select an intermediate scoring population for reducing potential variations in salivary flow rate. Food and drinks should not be consumed during saliva collection. Toda et al. reported that consuming food just prior to sampling can lead to false results of stress markers such as salivary cortisol. However, in some cases, food can be eaten $30 \mathrm{~min}$ to $1 \mathrm{~h}$ prior to spitting out the saliva [16]. The individual can rinse his/her mouth with deionized water and wait for at least $10 \mathrm{~min}$ before sample collection. Clear and readable labeling is necessary for proper sample identification, processing, and storage. Bar-coded labels or particulars written with permanent markers are mostly recommended for long-term storage [26].

"Saliva Collection Handbook" a guide issued by Salvimetrics (www.salvimetrics.com) justified certain measures to be considered while saliva sampling. Modern salivary assays are crafted to use small sample volumes (mostly less than $100 \mu \mathrm{L}$ ), and in many cases, stimulants are not needed to collect adequate sample volume. If stimulants are inevitable; it is advised to use very less amounts of it and in a consistent manner. Consider using visual or olfactory stimulation before an oral stimulant is given, practice the collection methodology with the study participants before starting the actual study to ensure that it is comfortable for them, and conduct a pilot study for ensuring that the oral stimulant does not cause any interference ("Saliva Collection Handbook," [41]).

Due to low concentrations and normal ranges of analytes in saliva compared with those in blood, there is a chance that blood contamination in saliva influences the levels of salivary biomarkers [24]. To avoid blood contamination in saliva, some recommendations can be considered: individuals must not brush their teeth at least 45 min prior to sample collection, no dental work to be done within $24 \mathrm{~h}$ prior to sampling, individuals must be screened for oral health injuries, and saliva samples that are visibly contaminated with blood is to be discarded and recollected ("Saliva Collection Handbook," [41]).

Another recommendation to be considered is to use appropriate collection methods for specific analyte. For example, some analytes can only be detected in samples which are collected by passive drool. Hence, using a swab to measure such analytes can result in underrecovery or over-recovery of the target analyte. Before sampling, it is good to follow certain practices such as avoiding foods with high acidity, sugar content, or high caffeine, since they may lower the $\mathrm{pH}$ and increase bacterial growth thereby affecting the assay; documenting consumption of caffeine, nicotine, alcohol, and prescribed/over-the-counter drug within $12 \mathrm{~h}$ prior to sampling and presence of oral diseases or injury and vigorous physical activity; avoiding eating a major meal at least $60 \mathrm{~min}$ prior to sample collection; rinsing the mouth to remove food residue; and waiting at least 10 min after rinsing to avoid sample dilution before sampling. Hence, following the guidelines and recommendations seriously will help an investigator or a medical laboratory technician to efficiently decipher the target analyte ("Saliva Collection Handbook," [41]). 


\section{FUTURE PERSPECTIVES}

Saliva sampling is a non-invasive procedure and has great potential to be used for studies aimed at analyzing time period, magnitude, and variation in immune responses and inflammation [49]. Being a patient-friendly diagnostic tool, saliva can be used to diagnose, monitor, or assess systemic inflammation in several chronic diseases such as tumors, infectious disease, hormone and drug level, hereditary diseases, serious conditions of anemia, and wound healing [45]. As saliva owns a list of advantages such as easy multiple sampling, stress-free and non-invasive (beneficial for infants, toddlers, and patients who face difficulty in providing blood samples), and reduction in healthcare costs as there is no need for professionals to carry out this work, it has got the potential to replace invasive diagnostic specimens such as blood and CSF. Correlation studies published in the past years clearly indicate that there is no much difference in the analyte concentrations in saliva when compared to blood, provided that saliva is collected, handled, and stored under the standard guidelines and recommendations. Transforming saliva to a traditional diagnostic specimen would create more opportunities in the field of biomedical research, especially in devising point-of-care equipment that can analyze the target analytes of patients. Notably, RTPCR analysis of inflammatory markers in saliva has got very limited reach in healthcare facilities, as mostly proteomic analysis of cytokines is performed in medical laboratories to diagnose the patient's condition. It is beyond doubt that proteomic assays provide a great deal of information about inflammation and other conditions, but upgrading the analysis to the gene level will help in extracting clear and meaningful information easily and much earlier than proteomic assays. With the popularization of molecular biology techniques such as RT-PCR, there seems to be a high chance for diagnosis and prognosis test to be shifted to genomic level of analysis for faster results [10]. Gene-level analysis in a non-invasive and stress-free diagnostic specimen such as saliva would be a breakthrough in the diagnostics field, easing the labor of laboratory technicians as well as reducing the stress in patients. Even though many studies were done using mRNA expression of inflammatory biomarkers worldwide, this technology is still not prevalent in clinical laboratories or in use as a point of care. This could be the lack of extensive validation and in-depth awareness of such a promising diagnostic method among healthcare innovators. Here, we highlight the potentiality of saliva as a diagnostic specimen as well as the use of modern-day highprecision molecular biology technique, RT-PCR, for early screening of inflammatory genes.

\section{CONCLUSION}

Saliva can be regarded as a potential specimen for detecting systemic inflammatory markers associated with active diseases, as most of these markers can be very well detected in saliva just as much as it is in the patient's blood. Currently, very limited studies targeting the detection of inflammatory biomarkers at the genetic level using RTPCR are reported. With due importance to early diagnosis and prognosis of diseases, this calls for members of the scientific community to perform as well as validate such studies being undertaken. Previous studies which attempted to use saliva as a diagnostic fluid were hampered by a lack of understanding of how the biomarkers enter saliva and ignorance in considering the guidelines or recommendations during sampling, handling, and storage of samples. These challenges have largely been met as a result of careful studies of salivary gland physiology, the different secretions that each gland produces, age and gender dependency of good quality saliva, storage conditions, development of sensitive detection methods like RTPCR, and educating the scientific and healthcare community on apt methods for collection, processing, and storage of salivary samples. With these advancements in the field, it paves the way to transforming saliva into a traditional diagnostic specimen which would create more opportunities in the field of biomedical research, especially in devising point-of-care equipment. The present review reveals the relevance of systemic inflammatory markers in saliva and its correlation with disease progression and intents to promote many more discoveries in the field of salivomics, with emphasis on the detection of genetic levels of inflammatory biomarkers using RT-PCR.

\section{ACKNOWLEDGEMENTS}

The authors would like to thank Zum Heilen Diagnostic \& Therapeutics Pvt. Ltd. for providing facilities and support to complete this work.

Data and Materials Availability. All data generated or analyzed during this study are included in this published article.

\section{AUTHOR CONTRIBUTION}

Nourin Shakeeb: Investigation, methodology, resources, writing - original drafting.

Prashanth Varkey: Conceptualization, project administration, review and editing. 
Amita Ajit: Conceptualization, methodology, writing - original drafting, investigation, resources, supervision, project administration, validation, visualization, original drafting, review and editing.

\section{DECLARATIONS}

We confirm that the manuscript has been read and approved by all named authors and that there are no other persons who satisfied the criteria for authorship but are not listed.

Ethics Approval and Consent to Participate. Not applicable.

Consent for Publication. The authors grant the publisher permission to publish the work in Inflammation.

Competing Interests. The authors declare no competing interests.

\section{REFERENCES}

1. Al-Rawi, N.H., F. Al-Marzooq, A.S. Al-Nuaimi, M.Y. Hachim, and R. Hamoudi. 2020. Salivary microRNA 155, 146a/b and 203: A pilot study for potentially non-invasive diagnostic biomarkers of periodontitis and diabetes mellitus. PLoS One 15 (8): e0237004.

2. Bhattarai, K.R., H.-R. Kim, and H.-J. Chae. 2018. Compliance with saliva collection protocol in healthy volunteers: Strategies for managing risk and errors. International Journal of Medical Sciences 15 (8): 823-831. https://doi.org/10.7150/ijms.25146.

3. Bonaventura, A., L. Liberale, A. Vecchié, M. Casula, F. Carbone, F. Dallegri, and F. Montecucco. 2016. Update on inflammatory biomarkers and treatments in ischemic stroke. International Journal of Molecular Sciences 17 (12). https://doi.org/10.3390/ijms17121967.

4. Boppana, S.B., S.A. Ross, M. Shimamura, A.L. Palmer, A. Ahmed, M.G. Michaels, P.J. Sánchez, D.I. Bernstein, R.W. Tolan Jr., Z. Novak, and N. Chowdhury. 2011. Saliva polymerase-chain-reaction assay for cytomegalovirus screening in newborns. New England Journal of Medicine 364 (22): 2111-2118. https://doi.org/10.1056/ NEJMoa1006561.

5. Byrne, M.L., N.M. O’Brien-Simpson, E.C. Reynolds, K.A. Walsh, K. Laughton, J.M. Waloszek, M.J. Woods, J. Trinder, and N.B. Allen. 2013. Acute phase protein and cytokine levels in serum and saliva: A comparison of detectable levels and correlations in a depressed and healthy adolescent sample. Brain, Behavior, and Immunity 34: 164-175. https://doi.org/10.1016/j.bbi.2013.08.010.

6. Chen, L., H. Deng, H. Cui, J. Fang, Z. Zuo, J. Deng, Y. Li, X. Wang, and L. Zhao. 2017. Inflammatory responses and inflammationassociated diseases in organs. Oncotarget 9 (6): 7204-7218. https://doi.org/10.18632/oncotarget.23208.

7. Cheng, Y., M. Pereira, N.P. Raukar, J.L. Reagan, M. Quesenberry, L. Goldberg, T. Borgovan, W.C. LaFrance Jr., M. Dooner, M. Deregibus, G. Camussi, B. Ramratnam, and P. Quesenberry. 2020.
Inflammation-related gene expression profiles of salivary extracellular vesicles in patients with head trauma. Neural Regeneration Research 15 (4): 676-681. https://doi.org/10.4103/16735374.266924.

8. Chiappin, S., G. Antonelli, R. Gatti, and E.F. De Palo. 2007. Saliva specimen: A new laboratory tool for diagnostic and basic investigation. Clinica Chimica Acta 383 (1): 30-40. https://doi.org/10.1016/ j.cca.2007.04.011.

9. Corey-Bloom, J., R.S. Fischer, A. Kim, C. Snell, G.M. Parkin, D.A. Granger, S.W. Granger, and E.A. Thomas. 2020. Levels of interleukin-6 in saliva, but not plasma, correlate with clinical metrics in Huntington's disease patients and healthy control subjects. International Journal of Molecular Sciences 21 (17). https://doi.org/ 10.3390/ijms21176363.

10. Desai, G.S., and S.T. Mathews. 2014. Saliva as a non-invasive diagnostic tool for inflammation and insulin-resistance. World Journal of Diabetes 5 (6): 730-738. https://doi.org/10.4239/ wjd.v5.i6.730.

11. Eagan, T.M.L., T. Ueland, P.D. Wagner, J.A. Hardie, T.E. Mollnes, J.K. Damås, et al. 2010. Systemic inflammatory markers in COPD: Results from the Bergen COPD cohort study. European Respiratory Journal 35 (3): 540-548.

12. Estévez, O., L. Anibarro, E. Garet, Á. Pallares, A. Pena, C. Villaverde, V. del Campo, and Á. González-Fernández. 2020. Identification of candidate host serum and saliva biomarkers for a better diagnosis of active and latent tuberculosis infection. PLoS One 15 (7): e0235859. https://doi.org/10.1371/journal.pone.0235859.

13. Fenoll-Palomares, C., J. Montagud, V. Sanchiz, B. Herreros, V. Hernández, M. Pérez, and A. Benages. 2004. Unstimulated salivary flow rate, $\mathrm{pH}$ and buffer capacity of saliva in healthy volunteers. Revista Española de Enfermedades Digestivas : Organo Oficial de La Sociedad Española de Patología Digestiva 96: 773-783. https:// doi.org/10.4321/S1130-01082004001100005.

14. Furman, D., J. Campisi, E. Verdin, P. Carrera-Bastos, S. Targ, C. Franceschi, L. Ferrucci, D.W. Gilroy, A. Fasano, G.W. Miller, A.H. Miller, A. Mantovani, C.M. Weyand, N. Barzilai, J.J. Goronzy, T.A. Rando, R.B. Effros, A. Lucia, N. Kleinstreuer, and G.M. Slavich. 2019. Chronic inflammation in the etiology of disease across the life span. Nature Medicine 25 (12): 1822-1832. https://doi.org/10.1038/ s41591-019-0675-0.

15. Gomes, M.A.B., F.H. Rodrigues, S.R. Afonso-Cardoso, A.M. Buso, A.G. Silva, S. Favoreto, and M.A. Souza. 2006. Levels of immunoglobulin A1 and messenger RNA for interferon gamma and tumor necrosis factor alpha in total saliva from patients with diabetes mellitus type 2 with chronic periodontal disease. Journal of Periodontal Research 41 (3): 177-183. https://doi.org/10.1111/j.16000765.2005.00851.x.

16. Granger, D.A., S.B. Johnson, S.L. Szanton, D. Out, and L.L. Schumann. 2012. Incorporating salivary biomarkers into nursing research: An overview and review of best practices. Biological Research for Nursing 14 (4): 347-356. https://doi.org/10.1177/ 1099800412443892.

17. Guerreiro, R.J., I. Santana, J.M. Brás, B. Santiago, A. Paiva, and C. Oliveira. 2007. Peripheral inflammatory cytokines as biomarkers in Alzheimer's disease and mild cognitive impairment. Neurodegenerative Diseases 4 (6): 406-412. https://doi.org/10.1159/000107700.

18. Hachim, M.Y., N.M. Elemam, R.K. Ramakrishnan, L. Salameh, R. Olivenstein, I.Y. Hachim, T. Venkatachalam, B. Mahboub, S. Al Heialy, R. Halwani, Q. Hamid, and R. Hamoudi. 2020. Blood and Salivary Amphiregulin Levels as Biomarkers for Asthma. Frontiers in Medicine 7: 561866. https://doi.org/10.3389/fmed.2020.561866. 
19. Heid, C.A., J. Stevens, K.J. Livak, and P.M. Williams. 1996. Real time quantitative PCR. Genome Research 6 (10): 986-994. https:// doi.org/10.1101/gr.6.10.986.

20. Hu, F.B., J.B. Meigs, T.Y. Li, N. Rifai, and J.E. Manson. 2004. Inflammatory markers and risk of developing type 2 diabetes in women. Diabetes 53 (3): 693-700. https://doi.org/10.2337/ diabetes.53.3.693

21. Hu, S., Y. Li, J. Wang, Y. Xie, K. Tjon, L. Wolinsky, R.R.O. Loo, J.A. Loo, and D.T. Wong. 2006. Human saliva proteome and transcriptome. Journal of Dental Research 85 (12): 1129-1133.

22. Il'yasova, D., L.H. Colbert, T.B. Harris, A.B. Newman, D.C. Bauer, S. Satterfield, and S.B. Kritchevsky. 2005. Circulating levels of inflammatory markers and cancer risk in the health aging and body composition cohort. Cancer Epidemiology, Biomarkers \& Prevention: A Publication of the American Association for Cancer Research, cosponsored by the American Society of Preventive Oncology 14 (10): 2413-2418. https://doi.org/10.1158/1055-9965.EPI05-0316.

23. John, M.A.S., Y. Li, X. Zhou, P. Denny, C.M. Ho, C. Montemagno, W. Shi, F. Qi, B. Wu, U. Sinha, and R. Jordan. 2004. Interleukin 6 and interleukin 8 as potential biomarkers for oral cavity and oropharyngeal squamous cell carcinoma. Archives of OtolaryngologyHead \& Neck Surgery 130 (8): 929-935.

24. Kang, J.-H., and H.-S. Kho. 2019. Blood contamination in salivary diagnostics: Current methods and their limitations. Clinical Chemistry and Laboratory Medicine (CCLM) 57 (8): 1115-1124. https:// doi.org/10.1515/cclm-2018-0739.

25. Kehm, R.D., J.A. McDonald, S.E. Fenton, M. Kavanaugh-Lynch, K.A. Leung, K.E. McKenzie, J.S. Mandelblatt, and M.B. Terry. 2020. Inflammatory biomarkers and breast cancer risk: A systematic review of the evidence and future potential for intervention research. International Journal of Environmental Research and Public Health 17 (15). https://doi.org/10.3390/ijerph17155445.

26. Kivlighan, K.T., D.A. Granger, E.B. Schwartz, V. Nelson, M. Curran, and E.A. Shirtcliff. 2004. Quantifying blood leakage into the oral mucosa and its effects on the measurement of cortisol, dehydroepiandrosterone, and testosterone in saliva. Hormones and Behavior 46 (1): 39-46. https://doi.org/10.1016/ j.yhbeh.2004.01.006.

27. Kralik, P., and M. Ricchi. 2017. A basic guide to real time PCR in microbial diagnostics: Definitions, parameters, and everything. Frontiers in Microbiology 8: 108. https://doi.org/10.3389/ fmicb.2017.00108.

28. MacRitchie, N., M. Frleta-Gilchrist, A. Sugiyama, T. Lawton, I.B. McInnes, and P. Maffia. 2020. Molecular imaging of inflammation-Current and emerging technologies for diagnosis and treatment. Pharmacology \& Therapeutics 211: 107550. https://doi.org/10.1016/j.pharmthera.2020.107550.

29. Malamud, D., and I.R. Rodriguez-Chavez. 2011. Saliva as a diagnostic fluid. Dental Clinics of North America 55 (1): 159-178. https://doi.org/10.1016/j.cden.2010.08.004.

30. Malathi, N., Mythili, S. and Vasanthi, H.R., (2014). Salivary diagnostics: A brief review. International Scholarly Research Notices, 2014.

31. Melguizo-Rodríguez, L., V.J. Costela-Ruiz, F.J. Manzano-Moreno, C. Ruiz, and R. Illescas-Montes. 2020. Salivary biomarkers and their application in the diagnosis and monitoring of the most common oral pathologies. International Journal of Molecular Sciences 21 (14): 5173.

32. Menon, M.M., R.V. Balagopal, K. Sajitha, K. Parvathy, G.B. Sangeetha, X.M. Arun, and J. Sureshkumar. 2016. Evaluation of salivary interleukin- 6 in children with early childhood caries after treatment. Contemporary Clinical Dentistry 7 (2): 198. https:// doi.org/10.4103/0976-237X.183059.

33. Menon, P.K., K. Kapila, and V.C. Ohri. 1999. Polymerase chain reaction and advances in infectious disease diagnosis. Medical Journal Armed Forces India 55 (3): 229-231.

34. Nam, Y., Y.-Y. Kim, J.-Y. Chang, and H.-S. Kho. 2019. Salivary biomarkers of inflammation and oxidative stress in healthy adults. Archives of Oral Biology 97: 215-222. https://doi.org/10.1016/ j.archoralbio.2018.10.026.

35. Oh, S.Y., S.-M. Kang, S.H. Kang, H.-J. Lee, T.-G. Kwon, J.-W. Kim, S.-T. Lee, S.-Y. Choi, and S.-H. Hong. 2020. Potential salivary mRNA biomarkers for early detection of oral cancer. Journal of Clinical Medicine 9 (1): 243. https://doi.org/10.3390/jcm9010243.

36. Out, D., R.J. Hall, D.A. Granger, G.G. Page, and S.J. Woods. 2012. Assessing salivary C-reactive protein: Longitudinal associations with systemic inflammation and cardiovascular disease risk in women exposed to intimate partner violence. Brain, Behavior, and Immunity 26 (4): 543-551. https://doi.org/10.1016/j.bbi.2012.01.019.

37. Park, J.-C., S.-H. Han, and I. Mook-Jung. 2020. Peripheral inflammatory biomarkers in Alzheimer's disease: A brief review. $B M B$ Reports 53 (1): 10-19. https://doi.org/10.5483/ BMBRep.2020.53.1.309.

38. Petersen, J.W., and G.M. Felker. 2006. Inflammatory biomarkers in heart failure. Congestive Heart Failure (Greenwich, Conn.) 12 (6): 324-328. https://doi.org/10.1111/j.1527-5299.2006.05595.x.

39. Prasad, S., A.K. Tyagi, and B.B. Aggarwal. 2016. Detection of inflammatory biomarkers in saliva and urine: Potential in diagnosis, prevention, and treatment for chronic diseases. Experimental Biology and Medicine 241 (8): 783-799. https://doi.org/10.1177/ 1535370216638770.

40. Roi, A., Rusu, L.C., Roi, C.I., Luca, R.E., Boia, S. and Munteanu, R.I., 2019. A new approach for the diagnosis of systemic and oral diseases based on salivary biomolecules. Disease markers, 2019. https://doi.org/10.1155/2019/8761860

41. Saliva Collection Handbook. (2017). Salimetrics. https:// salimetrics.com/saliva-collection-handbook/

42. Sapkota, D., T.M. Søland, H.K. Galtung, L.P. Sand, S. Giannecchini, To, K. K. W, M.C. Mendes-Correa, D. Giglio, B. Hasséus, and P.H. Braz-Silva. 2020. COVID-19 salivary signature: Diagnostic and research opportunities. Journal of Clinical Pathology. 74: 344-349. https://doi.org/10.1136/jclinpath-2020-206834.

43. Selvam, N.P., and J. Sadaksharam. 2015. Salivary interleukin-6 in the detection of oral cancer and precancer. Asia-Pacific Journal of Clinical Oncology 11 (3): 236-241.

44. Sharain, K., D. Hoppensteadt, V. Bansal, A. Singh, and J. Fareed. 2013. Progressive increase of inflammatory biomarkers in chronic kidney disease and end-stage renal disease. Clinical and Applied Thrombosis/Hemostasis: Official Journal of the International Academy of Clinical and Applied Thrombosis/Hemostasis 19 (3): 303308. https://doi.org/10.1177/1076029612454935.

45. S Sindhu, and Jagannathan, N., (2014). Saliva: a cutting edge in diagnostic procedures. Journal of Oral Diseases, 2014. https:// doi.org/10.1155/2014/168584

46. Singh, P., J.K. Verma, and J.K. Singh. 2020. Validation of Salivary Markers, IL-1 $\beta$, IL-8 and Lgals3bp for Detection of Oral Squamous Cell Carcinoma in an Indian Population. Scientific reports 10 (1): 19.

47. Slavich, G.M. 2015. Understanding inflammation, its regulation, and relevance for health: A top scientific and public priority. Brain, Behavior, and Immunity 45: 13-14. https://doi.org/10.1016/ j.bbi.2014.10.012.

48. Smith, C.H., B. Boland, Y. Daureeawoo, E. Donaldson, K. Small, and J. Tuomainen. 2013. Effect of aging on stimulated salivary flow 
in adults. Journal of the American Geriatrics Society 61 (5): 805808. https://doi.org/10.1111/jgs.12219.

49. Szabo, Y.Z., and D.C. Slavish. 2021. Measuring salivary markers of inflammation in health research: A review of methodological considerations and best practices. Psychoneuroendocrinology 124: 105069. https://doi.org/10.1016/j.psyneuen.2020.105069.

50. Thomadaki, K., E.J. Helmerhorst, N. Tian, X. Sun, W.L. Siqueira, D.R. Walt, and F.G. Oppenheim. 2011. Whole-saliva proteolysis and its impact on salivary diagnostics. Journal of Dental Research 90 (11): 1325-1330. https://doi.org/10.1177/0022034511420721.

51. Wang, B., L. Wu, J. Chen, L. Dong, C. Chen, Z. Wen, J. Hu, I. Fleming, and D.W. Wang. 2021. Metabolism pathways of arachidonic acids: Mechanisms and potential therapeutic targets. Signal Transduction and Targeted Therapy 6 (1): 1-30. https://doi.org/ 10.1038/s41392-020-00443-w.

52. Wang, T., X. Fu, Q. Chen, J.K. Patra, D. Wang, Z. Wang, and Z. Gai. 2019. Arachidonic acid metabolism and kidney inflammation. International Journal of Molecular Sciences 20 (15): 3683. https:// doi.org/10.3390/ijms20153683.

53. Wang, X., W. Bao, J. Liu, Y.Y. OuYang, D. Wang, S. Rong, X. Xiao, Z.L. Shan, Y. Zhang, P. Yao, and L.G. Liu. 2013. Inflammatory markers and risk of type 2 diabetes: A systematic review and meta-analysis. Diabetes Care 36 (1): 166-175.
54. Wei, F., C.-C. Lin, A. Joon, Z. Feng, G. Troche, M.E. Lira, D. Chia, M. Mao, C.-L. Ho, W.-C. Su, and D.T.W. Wong. 2014. Noninvasive saliva-based EGFR gene mutation detection in patients with lung cancer. American Journal of Respiratory and Critical Care Medicine 190 (10): 1117-1126. https://doi.org/10.1164/ rccm.201406-1003OC.

55. Yi, L., H. Pimentel, N.L. Bray, and L. Pachter. 2018. Gene-level differential analysis at transcript-level resolution. Genome Biology 19 (1): 53. https://doi.org/10.1186/s13059-018-1419-z.

56. Yoshida, R. A., Gorjão, R., Mayer, M. P. A., Corazza, P. F. L., Guare, R. O., Ferreira, A. C. F. M., \& Santos, M. T. B. R. 2019. Inflammatory markers in the saliva of cerebral palsy individuals with gingivitis after periodontal treatment. Brazilian Oral Research 33.

57. Zakynthinos, E., and N. Pappa. 2009. Inflammatory biomarkers in coronary artery disease. Journal of Cardiology 53 (3): 317-333. https://doi.org/10.1016/j.jjcc.2008.12.007.

58. Zielińska, K., Karczmarek-Borowska, B., Kwaśniak, K., CzarnikKwaśniak, J., Ludwin, A., Lewandowski, B., \& Tabarkiewicz, J. (2020). Salivary IL-17A, IL-17F, and TNF- $\alpha$ are associated with disease advancement in patients with oral and oropharyngeal cancer. Journal of Immunology Research, 2020. https://doi.org/10.1155/ 2020/3928504

Publisher's Note Springer Nature remains neutral with regard to jurisdictional claims in published maps and institutional affiliations. 